\title{
Single screw extrusion control - A comprehensive review and directions for improvements
}

DOI:

10.1016/j.conengprac.2016.03.008

\section{Document Version}

Accepted author manuscript

Link to publication record in Manchester Research Explorer

\section{Citation for published version (APA):}

Abeykoon, C. (2016). Single screw extrusion control - A comprehensive review and directions for improvements. Control Engineering Practice, 51, 69-80. https://doi.org/10.1016/j.conengprac.2016.03.008

\section{Published in:}

Control Engineering Practice

\section{Citing this paper}

Please note that where the full-text provided on Manchester Research Explorer is the Author Accepted Manuscript or Proof version this may differ from the final Published version. If citing, it is advised that you check and use the publisher's definitive version.

\section{General rights}

Copyright and moral rights for the publications made accessible in the Research Explorer are retained by the authors and/or other copyright owners and it is a condition of accessing publications that users recognise and abide by the legal requirements associated with these rights.

\section{Takedown policy}

If you believe that this document breaches copyright please refer to the University of Manchester's Takedown Procedures [http://man.ac.uk/04Y6Bo] or contact uml.scholarlycommunications@manchester.ac.uk providing relevant details, so we can investigate your claim.

\section{OPEN ACCESS}




\title{
Single Screw Extrusion Control: A Comprehensive Review and Directions for Improvements
}

\author{
Chamil Abeykoon \\ ${ }^{a}$ The Faculty of Engineering and Physical Sciences, The University of Manchester, Oxford Road, Manchester, M13 9PL, United Kingdom
}

\begin{abstract}
Polymer extrusion is usually a complex process, particularly due to the coupled nature of process parameters, and hence highly prone to fluctuations. Although a number of different approaches have been attempted in research/industry over the last few decades for extrusion control, it is still experiencing some problems in achieving consistent product quality. Presently, most of the polymer processing extruders are equipped with PID controllers mainly for the control of the screw speed and barrel temperatures in their set limits. It seems that only both of these controllers are commonly used as the major aids of process control to achieve the required melt quality. Although, the quality of the melt output (i.e., a thermally homogeneous melt output which is constant in quantity and quality over the time) is the key variable in polymer extrusion, only a few control techniques are available which make control decisions by observing the actual melt flow quality. Therefore, the development of new control strategies which consider the actual melt quality, perhaps incorporating industrially popular nonlinear techniques such as artificial intelligence, should be highly valuable. In this work, a critical evaluation is made on the state-of-the-art of the previous control approaches in polymer extrusion in industry and research while identifying their limitations. Then, some of the possible directions for future research and also to develop an advanced process control strategy are presented by eliminating a few of the existing limitations.
\end{abstract}

Keywords: Polymer extrusion, Melt quality, Process monitoring, Modelling, Soft sensing, Process control, Fuzzy logic.

\section{INTRODUCTION}

It is obvious that the global energy demand has been increasing rapidly over the last few decades. Meantime, it has been predicted that there will be a scarcity of available energy sources in the world within the next few decades and hence the energy prices are continually increasing [1]. In general, it is well-known that a significant portion of the global annual energy production is consumed for the transportation purposes [2] and hence scientists are exploring ways and means to cut down the energy usage particularly in the transport sector. Here, the replacement of the conventional raw materials such as metal, glass, wood, etc with the advanced polymeric materials with a high strength to weight ratio has been becoming a one of the potential solutions. In addition to the significant reduction of weight, polymeric materials offer a number of other advantages such as ease of forming into complex shapes, high temperature/chemical/impact resistance, high clarity, re-processability, low cost, etc over other materials. Therefore, polymeric materials have become widely popular not only in the transport sector but also in construction, medical, electrical and electronic, household applications as well. Under these circumstances, polymer processing techniques are becoming highly important production methods in the industry. Among the polymer processing techniques, extrusion can be recognized as one

Email address: chamil . abeykoon@manchester.ac.uk; yabeykoon01@qub.ac.uk; phone: +441613062540 (Chamil Abeykoon) of the fundamental methods and hence the improvements of the process monitoring and control will be highly useful for catering the growing demand with good quality products at low unit cost. Moreover, such improvements should help to minimize the process related common problems such as long downtimes, waste of material, energy, labor, etc which offer several economic benefits.

Polymer extrusion is usually involved in the final production of many polymer products such as pipes, films, sheets, tubes, rods, etc. It is also an intermediate processing stage in injection moulded, blown film, thermoformed, and blow moulded products. An extruder is a machine which processes material by conveying it along a screw and forcing it through a die at a certain pressure. The screw is the key component of an extruder and it has been divided into three main functional/geometrical zones (i.e., solids conveying or feed, melting or compression, and metering or pumping) which are generally based on the primary operations of an extruder as shown in Figure 1. The feedstock material fed into the machine through the hopper is conveyed along the screw while absorbing the heat provided by the barrel heaters and through process mechanical work. Eventually, a molten flow of material is forced into the die which forms the material into the desired shape. In general, the main function of an extruder is to deliver a homogeneous, well mixed polymer melt at specified uniform temperature and pressure. In order to accomplish this requirement, extruders are generally equipped with an efficient drive and a feed system, a screw designed to melt and convey the polymer and devices such as temperature and pressure sensors which are required to monitor 


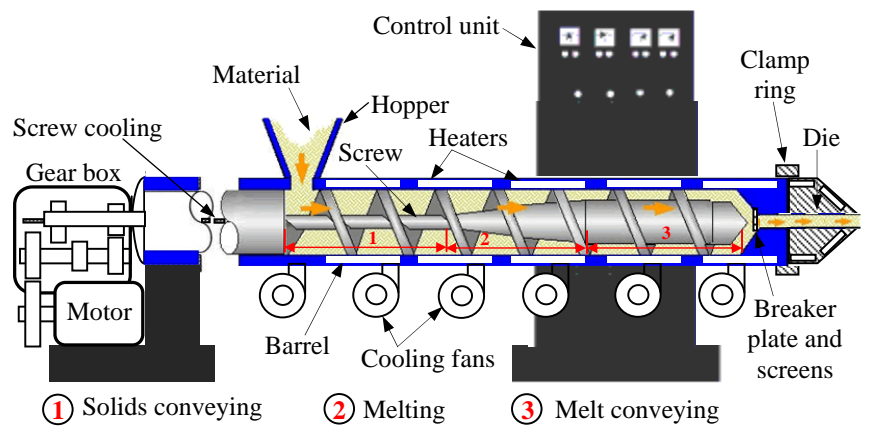

Figure 1: Basic components of a single screw extruder

the system for troubleshooting and control. More details on the process operation and mechanisms of polymer extrusion can be found in the literature $[3,4,5]$.

In spite of the significant improvements in polymer extrusion field over the last few decades, process thermal monitoring and control still remain an issue. Process operators have to face challenges in achieving the required quality of the melt output (e.g., melt flow thermal stability/homogeneity, consistency of the flow rate, etc) with prevailing knowledge and technology. For example, achieving good thermal stability is a key to form high quality extruded products as thermal fluctuations may cause to generate problems in physical and mechanical properties of the products. Therefore, continuous and accurate monitoring of the major process variables and also the use of advanced process control strategies are highly invaluable to form good quality melt output.

\subsection{Importance of improved process monitoring and control}

The key idea behind the process monitoring is to identify inherent process problems and to develop strategies to control them, both through machine design and process operation. Obviously, the performance of the process control strategies depends on the accuracy and the quality of the process monitoring techniques (i.e., depends on how good process problems are identified and understood). An accurate control cannot be achieved if the process cannot be monitored accurately [6]. Overall, process control relates to the selection and tuning of processing conditions to maintain the process efficiency and product quality for a specific material and a machine. In fact, the main objective of any process controller is to achieve good quality products while achieving a better process efficiency in terms of the use of material, energy, labour, time, etc.

In polymer extrusion, melt output from the machines should be uniform throughout the process (i.e., the uniformity of the melt temperature over the time and temperature homogeneity across the melt flow) to achieve good quality products. Temporal thermal variations of melt output may cause to generate variations of melt pressure resulting in output rate variations, non-uniformities of optical/mechanical/chemical properties of the extruded parts, extrudate with un-melted particles, etc [7]. In fact, process problems can occur due to the variability of processing materials [8, 9, 10], machine geometry $[11,12,13,14,15]$ and process settings [4, 8, 13, 16, 17]. Obviously, the processing problems attributed to the machines' functionality (e.g., screw misalignments, vibrations of machine parts, inaccuracies of screw design) have to be addressed at the stage of machine design and the selection of optimum operating conditions may be the most important factor to achieve process requirements for a given machine and a material. In such a situation, having a process controller which can accurately detect and control process problems is invaluable.

Although the quality of the melt output seems to be the major consideration in polymer extrusion, it seems that no many control techniques are available which make control decisions by observing the actual melt flow quality. This may be due to the practical difficulties of monitoring the melt quality across the flow cross-section without disturbing the melt flow. Presently, most of the polymer processing extruders are equipped with speed controllers (i.e., to maintain the screw speed within set limits) and temperature controllers (i.e., to maintain the barrel zone temperatures within their set limits). Both of these controllers are commonly used as the major aids of process control to achieve the required melt quality. The feedback for speed control is obtained via instruments such as tachometer generators (i.e., a device which can convert the measured speed into an analog voltage signal) while temperature feedback is usually obtained from the thermocouples attached to the extruder barrel/die wall. In fact, the availability of a controller which makes necessary control decisions by observing the actual quality of the melt (by using an appropriate measurement technique) would be highly useful rather than maintaining the barrel set temperatures and/or screw speed within their set limits without obtaining any feedback on the actual melt quality. Based on this background, further research to improve the process control in polymer extrusion was timely and hence this study was mainly focused on exploring possible directions for possible improvements. Initially, a critical evaluation of the previous extrusion control approaches are presented while identifying their capabilities and limitations. Then, the current limitations relating to the extrusion control are discussed while proposing possible directions for developing advanced control approaches for polymer extrusion control.

\subsection{Approaches in polymer extrusion control}

\subsubsection{Melt temperature and/or pressure control}

Control schemes based on temperature and/or pressure have been prevalent since the late 1970s. Several researchers attempted to control the melt temperature and pressure as an indirect approach of controlling the melt viscosity due to the difficulties encountered in real-time viscosity monitoring. Melt temperature control helps to avoid viscosity fluctuations (for a consistent feed material) and thermal degradation of the material. Stable pressure generation ensures a consistent mass flow rate. Initially, several researchers used linear Laplace transform techniques to develop control schemes relating to extrusion melt temperature and pressure via proportional-integralderivative (PID) algorithms. Later, some other researchers used time series techniques for temperature and pressure control, which are capable of developing more sophisticated models allowing identification and control of process disturbances. 


\section{a. Control schemes based on Laplace transform or time series models}

The first study on the dynamic extruder pressure was carried out by White and Schott [18] in early 70s. Step and pulse tests of the screw speed were performed and identified a second order transfer function to represent the extruder by using a Laplace domain representation of the input-output relationship. The developed transfer function presented the extruder mass flow rate in terms of screw speed and feed material changes. Also, a step test of the feed material (i.e., by changing the feed material) was carried out to determine the extruder residence time and proposed that the control of melt pressure in the melt conveying zone and/or the die would control the extruder output rate.

Fontaine [19] developed Laplace transfer function models for melt throughput, exit pressure and temperature to changes in the back pressure valve setting, barrel heater power and screw speed. A digital filtering method was used to filter the fluctuations in the observed process signals rather than trying to control them. Then, a proportional-integral (PI) controller was proposed for the control of die and adapter temperatures based on the filtered process signals. Screw speed step changes (i.e., 8.15 rpm changes from an $85 \mathrm{rpm}$ screw speed) were used to verify the performance of the control algorithm. However, the usefulness of the proposed controller was limited as process signals were pre-filtered to remove some of the inherent fluctuations without considering the relations of these fluctuations with the product quality.

Fingerle [20] attempted to control melt temperature by introducing a special extruder modification. An annular section of adjustable length was added at the end of the extruder barrel, adjusting the length of the section was used to control the rate of viscous dissipation in the extruder. The responses of melt temperature and pressure to step changes in screw speed and the annular section length were identified. Melt temperature was found to be proportional to the length of the annulus but the annulus length did not influence the pressure at the die. A first order dead time temperature response were proposed to both screw speed and length, and a second order pressure response to screw speed. A controller was implemented with a PID algorithm and gave good temperature control in comparison with temperature control via heat transfer through the barrel. However, this control method involves complex modifications to the extruder which may not industrially possible and hence may limit its practical use.

Dormeier [21] proposed a PID control algorithm to regulate melt temperature of an extruder with three heating zones. The third zone heater input was used as the manipulated variable, and step changes applied to the second zone heater were used as disturbances for model identification. The controller was effective in regulating long-term disturbances but was not good at regulating high frequency disturbances.

Muhrer et al. [22] proposed a cascade PI control scheme to control the melt temperature by manipulating barrel zone temperatures. Extruder dynamics were presented by a first order plus time delay model. The proposed controller was imple- mented on an extruder and its disturbance rejection capability was tested by adding feed material property changes. The results showed that the controller was good in reducing the effects of process disturbances on the melt temperature. Moreover, the authors stated that the controller tuning has to repeat as changing the process operating conditions while re-determining the appropriate process gains (i.e., proportional and integral gains of the controller).

Chan and Lee [23], Chan et al. [24] and Nelson et al. [25] proposed Laplace transfer function models (e.g., a first order, a second order and a lead-lag) to describe dynamic responses of an extrusion process. Laplace transfer function models accounted for the basic type of temperature and pressure responses with fitting of a phenomenological kinetic-elastic model to calculate other required parameters. The kinetic portion of the model accounted for the nonlinearities associated with structural changes of materials while the elastic portion incorporated with the elastic behaviour of the polymer solution or melt. The kinetic-elastic model was found to be adequately describe the dynamic pressure responses of the process but it did not account for different responses caused by different magnitudes of screw speed step changes. Also, the model assumes isothermal processing conditions and requires detailed knowledge of material rheological properties for its use in process control.

Parnaby et al. [26] and Kochhar and Parnaby [27] modelled the extrusion process parameters by using a time series technique. Linearised models relating to small perturbations of the screw speed were identified by observing corresponding changes of the melt pressure and temperature at the die. A control scheme was proposed to control the melt temperature by manipulating the screw speed while melt pressure and flow rate were controlled by manipulating the die restrictor valve setting (i.e., not a common feature in extrusion). Simulations were carried out to calculate the control actions necessary to correct for drifting temperature and stated that the melt temperature predicted by the model was quite close to the measured temperature. Moreover, the authors encouraged to use feed forward or predictive control for extrusion process control based on their results. Later, Hassan and Parnaby [28] used an adaptive control approach to develop a model reference steadystate computer controller. A steady-state model was identified from linear regression of plant data to determine the desirable melt temperature and pressure to produce the required output. The process operator had to decide only the desired output and upper melt temperature limit, the rest of the control variable set points were continually set in-line by a pre-programmed computer. Process errors were identified by the cascade controller and manipulated the screw speed, restrictor valve angular position and barrel wall set temperatures via slave servomechanisms to achieve the required plant output. Changes to the wall temperature set points were implemented by a PID algorithm. The authors claimed that the controller was able to maintain the melt temperature within $\pm 1 \%$ of the required limits.

Costin et al. [29] used a time series technique to develop dynamic transfer function models between the screw speed and 
pressure and also to model the disturbances associated with the extruder pressure. A digital PI and self-tuning regulator (STR) algorithms were implemented to regulate the pressure by manipulating the screw speed. A number of screw speed step tests were performed to estimate process time constant and to determine the system linearity while various filters were used to eliminate the signal noise. Filtering of the signal noise was quite difficult and the implementation of the filter resulted in changing of the process transfer function and hence affected the controller performance. A combination of the STR and a filter failed to control the process and the PI algorithm with a filter gave relatively good results.

Germuska et al. [30] proposed an adaptive and multivariable control approaches to control the melt pressure, melt temperature and extrudate thickness by manipulating the screw speed, die heater power and the mass flow rate. The time series and Laplace transfer function models were used to implement a control scheme incorporating Dahlin feedback controllers (see [31] for details on Dahlin controllers) to control the melt pressure and temperature in order to maintain melt viscosity and extrudate thickness to achieve product dimensional accuracy. The proposed control scheme showed good steady-state control for changes to melt pressure and temperature set points although the extrudate thickness controller did not perform well due to noisy thickness measurements.

\section{b. Control schemes based on artificial intelligence techniques}

Although artificial intelligence (AI) techniques are still less popular in polymer processing industry, these are highly popular in some of the current industrial applications such as process control in other industries (e.g., temperature, pressure and level control, failure diagnosis and distillation column control); house hold applications like washing machines and refrigerators; motor control in power industry; various automobile, robotics and aerospace applications, etc [32].

Taur et al. [33] proposed a fuzzy PID temperature control for extruder barrel temperatures with if-then rules and triangular shaped membership functions which exhibited good control capabilities. Tasi and Lu [34] developed a single-loop fuzzy supervisory predictive PID controller also for extruder barrel temperature control. PID gains were estimated using a generalised predictive control technique. A real-time algorithm was applied to achieve control actions incorporating PID and fuzzy supervision. The controller set-point tracking performance was verified by experiments and successful results were achieved with steady state errors of $\pm 0.4^{\circ} \mathrm{C}$ and a small overshoot. Yusuf et al. [35] used fuzzy genetic algorithms in extruder barrel temperature control. The optimum shapes for membership functions of a fuzzy logic controller (FLC) were found using a genetic algorithm (GA). Simulation results showed that the optimised controller gave a much faster settling time with no overshoot. Ravi and Balakrishnan [36, 37] used AI techniques in extruder barrel temperature control. The results showed that a FLC can perform well with a lesser overshoot than a PI controller. Another work carried out by them [38, 39] found that a neurofuzzy controller gave better performance than fuzzy logic and PID controllers in extruder barrel temperature control. In fact, all of these studies based on AI techniques have focused only on the control of the barrel set temperatures in their set limits and did not attempt to control the melt temperature.

\section{c. Control schemes based on other techniques}

Menges and Meissner [40] proposed three control schemes to control the melt temperature of an extruder at the entrance of the die:

(i). A cascade control scheme

(ii). A control scheme based on the heating energy at the last barrel temperature zone

(iii). A control scheme based on the frictional energy which was based on adjusting a conical shaped valve in front of the screw tip.

A sensor which can make four radial measurements across the melt flow (based on the method proposed by Leeuwen [41]) was used for melt temperature measurements. It was mentioned that the first and the third control schemes gave satisfactory results. Of these two control schemes, the third scheme gave much faster responses but was not industrially compatible as they used a conical valve, which was not a usual industrial device, to control the melt flow. Moreover, the melt temperature measurement technique used in this work was also undesirable for industrial applications as it was highly disturbing the melt flow.

Dastych et al. [42] proposed a robust multi-input-multioutput (MIMO) control and a decentralised discrete model reference adaptive control for an extruder. Extrudate melt temperature and the temperature profile along the extruder were controlled by manipulating corresponding heaters and the screw speed. The MIMO controllers' $3 \times 3$ model was based on a linear-quadratic-Gaussian/loop-transfer-recovery (LQG/LTR) method and it controlled melt temperature by manipulating the power of the third and fourth barrel zones' heaters. The adaptive controller was based on their previous work [43] and it controlled the set temperatures of the extruder barrel zones and melt temperature. The performance of both controllers was verified by simulation and the results showed that the controllers can handle uncertainties and time varying or load depending plant parameters better than typical PID controllers.

Mercure and Trainor [44] manipulated extruder barrel set temperatures to control the melt temperature by using PID algorithms. The time dependent partial differential equations were used to formulate a first principle mathematical model of the extruder barrel temperature. The heat required for polymer melting was assumed to be negligible compared to the heat loss by convection while making a few other assumptions on barrel heat transfer and heat losses. The model parameter estimation and the optimisation of the controller tuning parameters were performed by virtue of software packages. The controller performance was verified by simulation and the authors claimed that it offered a number of advantages (e.g., reduced settling time, automatic start-up and reduced down time) over conventional controllers. However, the controller performance might be affected by simplifying assumptions and the difficulties of selecting the exact boundary conditions for solving differential equations (i.e., boundary conditions may vary with time, tem- 
perature, materials, screw speed, etc).

$\mathrm{Ng}$ et al. [45] proposed a discrete control strategy for an extruder gear pump arrangement to control the extruder pressure. The controller was designed based on a discrete optimal regulator solution and also included automatic dead time compensation. The sampling time of the controller was decided from an automatic sampling time selection algorithm which was based on an inter-sample behaviour. The controller performance was evaluated by simulation and the authors stated that it gave improved set-point tracking performance and disturbance rejection capability than an existing PI controller.

Lin and Lee [46] identified extrusion plant dynamics by a least squares approximation associated with loss and covariance functions, applied to a set of experimental data. The authors used the data from Parnaby et al. [26] and identified a single-input-single-output (SISO) third order state-space dynamic model. An integral observer control methodology was proposed incorporating the proposed dynamic model, and the extruder screw speed was manipulated to control the melt pressure and temperature. The controller was implemented by using software packages (e.g., Matlab and Mathematica) and the simulation results showed that the control of pressure and temperature in the limits of $\pm 0.5 \mathrm{MPa}$ and $\pm 2.5^{\circ} \mathrm{C}$, respectively.

Previdi et al. [47] proposed a prototype feedback controller for melt temperature and pressure of an extruder. Real-time regulation of temperature and pressure was achieved via two independent outer SISO control loops (i.e., for output temperature and pressure) and seven identical inner SISO control loops for barrel heater temperature control. Each control loop was implemented with a classical PID structure. The control scheme was implemented on a laboratory extruder and tested by applying step changes in the set-point of the output pressure ( 3 bar steps), output temperature $\left(10^{\circ} \mathrm{C}\right.$ steps) and material (i.e., by changing the material density and material type). The results obtained were better than the results obtained from a pump-equipped extruder which did not have feedback control. In conclusion, the authors stated that the proposed control system is a costeffective alternative for volumetric mechanical pumps while it is also a robust and an accurate solution to extrusion process control within required tolerances.

Recent work by Jiang et al. [48] proposed a SISO generalized predictive control (GPC) of the melt pressure at die output and a MIMO GPC to barrel set temperatures. A pressure model was identified via system identification to predict future output of the controlled variable. Also, the structure of the barrel temperature process model was obtained through theoretical analysis and calculation. The results of the melt pressure controller tracked the set point precisely. For the barrel set temperature, a feed-forward controller was incorporated into the multi-variable generalized predictive control (MGPC) to compensate for the disturbance caused by the change in melt pressure changes. The efficacy of the proposed control system was verified quantitatively using the product diameter as the quality measurement and it was outperformed over commercially available PID controllers.

\subsubsection{Viscosity control}

Revesz and Hubeny [49] proposed a closed loop viscosity control scheme incorporating a torque sensor based viscometer. The torque function observed was included two main components: a viscosity-dependent component and a speed-dependent component. The approach was to maintain the viscositydependent component at a desired value by compensating the speed-dependent component. A cascade control was proposed to the PID controllers used in barrel temperature control. However, the control scheme was not verified either by simulation or experiment due to some instrumentation problems.

Chiu and Lin [50] used an auto-regressive-moving-average (ARMA) model to investigate process disturbances and proposed a constrained minimum variance (CMV) closed-loop controller to reduce the melt viscosity variations. An in-line viscometer was designed and used to measure the viscosity of polymer melts between the screw end and the die. A combination of a plant model and a disturbance model which were obtained experimentally was used to develop the controller. The screw speed was manipulated to control the melt viscosity under constant temperature conditions. Simulation and experimental results showed $39.1 \%$ and $24.3 \%$ reductions of viscosity variance respectively with the CMV controller than an open loop control method. However, the size of the screw speed step change (i.e., $300 \mathrm{rpm}$ ) used for simulation was unrealistic and also the reasons for the difference between experimental and simulation results were not discussed. Another work by Chiu et al.[51] and Chiu and Pong [52, 53] introduced a fuzzy gainscheduled PID controller (incorporating an in-line viscometer) to control the melt viscosity by manipulating the screw speed. A second order model related to the viscosity and screw speed was empirically developed (i.e., from plant step responses) to approximate the extruder dynamics. The desired PID gains, response time and fuzzy rules were derived based on the process responses to the screw speed step changes. The results obtained from the fuzzy-PID controller showed a lesser overshoot and a faster settling time than a PID controller tuned by the Ziegler-Nichols method. Overall, Chiu and colleagues attempted a number of different strategies to control the melt viscosity based on in-line rheometer measurements as discussed above. Both ARMA and fuzzy logic techniques were applied but the investigations were limited in scope. Screw speed was used as the only manipulating variable. Both model development and testing were limited to a singular step change at a fixed operating point; these were not realistic of input manipulation in practical operation. Also, they have encountered some problems with instrumentation which affected the performance of the proposed controllers.

Kumar et al. [54] proposed an inferential technique for online viscosity estimation and closed-loop control for a polymer compounding process which involves blending of two polymers. A physics-based dynamic model was developed which describes the dynamic effects of common process inputs (e.g., raw material feed rates, screw speed) on measured outputs (e.g., die pressure). The model was coupled with on-line viscosity estimation in a wide range of operating conditions including raw 
materials and composition changes. The accuracy of viscosity estimation depends on the accuracy of the on-line measurements of feed rates and the die pressure. The estimated viscosity values were within the range of $\pm 10 \%$ to the off-line measurements made by a capillary rheometer. The controller was aimed to reject the effects of disturbances (e.g., raw material variations) or to track changes in viscosity set point for grade changes with the same basic raw materials. A PI controller with first order response was proposed to manipulate the feed composition to maintain the estimated viscosity at the set point. The control scheme was implemented on a laboratory extruder and tested with unknown raw material changes. The required feed composition was decided by using ratio control logic to vary the individual material federates while maintaining the desired output. The authors stated that the controller performed well over unknown disturbances and its efficacy depended on the accuracy of viscosity estimation.

Several authors proposed viscosity control strategies $[55,56$, 57] and also some other control approaches [58, 59] for reactive extrusion which mainly uses an extruder as a chemical reactor. Reactive extrusion differs from conventional polymer extrusion methods, where synthesis is a separate operation and the extruder serves only as a processing aid. As a result, most of the control schemes designed for reactive extrusion process may not be compatible with conventional continuous single screw extruders and hence not discussed in this paper.

\subsubsection{Other control approaches}

Stevenson [60] used a statistical technique to identify sources of fluctuations in the operation of extrusion lines. Cross correlations among process variables (i.e., extrudate layer thickness, weight, flow rate, surface temperature and line tension; screw speed; barrel pressure) were used to estimate offset times and to identify variables that were significantly correlated. These correlations were compared to the expected correlation patterns of usual process fluctuations. However, there is no clear mention on how the expected patterns of process fluctuations were determined. The author stated that the primary cause of shortterm fluctuations in extruders is the throughput surging. Moreover, he claimed that this study formed the basis for a novel process controller to minimise the consequences of short-term variations in extruder's output.

Yang and Lee $[61,62]$ proposed an analytical flow model for on-line control of profile extrusion. The proposed model was used to calculate the effects of the die wall temperature on the polymer melt flow. A dual single-loop feedback PI controller and a multivariable feed forward controller were implemented (based on two different algorithms) to compensate for set point changes of extrudate shape and size, and load disturbances. The extrudate size and shape were controlled by using the take-up speed and the die wall set temperature, respectively. The authors stated that the controllers were relatively good only for controlling long-term disturbances.

Guo et al. [63] proposed a FLC which included two interactive schemes (i.e., active pattern analysis and adaptive fuzzy control schemes). The pattern analysis scheme recognised the current operating state (i.e., pattern) of the process. Once the pattern was identified, the required control actions were selected by the strategy selection model. The adaptive fuzzy control scheme consisted of three sub-models associated with different control objectives and these adjusted the fuzzy rules and membership functions according to the dynamic behaviour of the process. The controller was capable of maintaining the product dimensions more stably within the required range while rejecting the external disturbances rapidly.

Ohshima and Tanigaki [64] proposed a quality control system for polymer production plants, which integrates optimal control with on-line sensing and scheduling techniques. Report by Kano and Ogawa [65] has provided a detailed description on the state-of-art of the control of industrial processes including the use of soft approaches in process control. As they claimed, 'for the polymer production industries, the competitive edge will come from the technology that excels in controlling the polymer properties in a consistent way over the entire plant and in maximizing the production performance while keeping safety regulations'.

Jing et al [66] proposed new real-time energy monitoring methods without the need to install power meters or to develop data-driven models. The effects of process settings on energy efficiency and melt quality were studied based on developed monitoring methods. Then, a fuzzy logic controller was developed for a single screw extruder to achieve good melt quality. The resultant performance of the developed controller showed it to be a satisfactory alternative to the expensive gear pump. Also, they stated that the energy efficiency of the extruder can further be achieved by optimising the temperature settings.

A few other studies available in the literature have presented techniques on process optimization and quality control together with some useful information on polymer extrusion [67, 68, 69, $70,71,72,73,74,75]$.

\section{Discussion}

A summary of the existing major control approaches for continuous single screw extrusion (discussed in the above) is given in Table 1. Here, the abbreviations, LT, TS and GA stand for the Laplace transform, time series and genetic algorithm, respectively.

\subsection{Limitations of the existing extrusion control}

Varying degrees of success have been achieved in the area of polymer extrusion process control by the manipulation of the barrel set temperatures and screw speed using empirical modelling techniques. The control of melt quality and throughput has traditionally been attempted by joint regulation of melt pressure (i.e., for mass throughput stability) and melt temperature (i.e., for melt thermal homogeneity). In fact, the temperature and pressure control methods in polymer extrusion seem to be well established than the other parameter control schemes. Moreover, the melt viscosity control has also been considered by a number of researches whilst some work attempted for alternative approaches (i.e., apart from the temperature, pressure and viscosity) in process control. Overall, the majority 
Table 1: Summary of the existing major control approaches for continuous single screw extrusion

\begin{tabular}{|c|c|c|c|c|}
\hline \multicolumn{5}{|c|}{ Temperature and/or Pressure control } \\
\hline No & Author/s-Year & Model type & Control method & \begin{tabular}{|l|l|} 
Comments on the controller performance/application \\
\end{tabular} \\
\hline 1 & Fontaine [19]-1975 & LT & PI & pre-filtering of signals may have influenced adversely on the controller's performance \\
\hline 2 & Fingerle $[20]-1978$ & & PID & an annular section of adjustable length was used which may limit the controller's practical use \\
\hline 3 & Dormeier [21]-1979 & LT & PID & was good for controlling long-term disturbances but was not good for short-term disturbances \\
\hline 4 & Muhrer et al. [22]-1983 & LT & Cascade PI & controller's tuning should be repeated for different process operating conditions \\
\hline 5 & Chan and Lee [23]-1984, Chan et al. [24]-1986, Nelson et al. [25]-1986 & LT & & assumed isothermal processing conditions, needs the knowledge of material rheological properties \\
\hline 6 & Parnaby et al. [26]-1975,Kochhar and Parnaby [27] -1977 & TS & PID & used a die restrictor valve which may limit the controller's practical use \\
\hline 7 & Hassan and Parnaby $[28]-1981$ & Linear Regression & Cascade PID & used a die restrictor valve which may limit the controller's practical use \\
\hline 8 & Costin et al. [29]-1982 & TS & PI, Self-tuning regulator (STR) & a STR with a filter was not good but a PI controller with a filter was good in performance \\
\hline 9 & Germuska et al. [30]-1984 & LT and TS & Dahlin & had problems with noisy measurements \\
\hline 10 & Taur et al. [33]-1995, Tasi and Lu [34]-1998 & if-then rules & Fuzzy PID & to control the barrel set temperature, was better than a PID controller \\
\hline 11 & Yusuf et al. [35]-2010 & if-then rules & Fuzzy logic with a GA & to control the barrel set temperature, was better than a PID controller \\
\hline$\frac{11}{12}$ & Ravi and Balakrishnan $[36,37]-2010,2011$ & if-then rules & Fuzzy logic & to control the barrel set temperature, was better than a PI controller \\
\hline 13 & Ravi and Balakrishnan $[38,39]-2010,2011$ & 11-ün тиеs & Neuro-Fuzzy & to control the barrel set temperature, was better than a PID controller and a FLC \\
\hline 14 & Menges and Meissner [40]-1972 & & Cascade PID & temperature measurement technique and the conical value were not industrially compatible \\
\hline 15 & Wiemer and Unbehauen [43]-1987, Dastych et al. [42]-1988 & Linear-Quadratic-Gaussian & Adaptive & was better than a PID controller \\
\hline 16 & Mercure and Trainor [44]-1989 & Partial differential & PID & first principle models were based on the several assumptions which may lead to poor control performance \\
\hline 17 & Ng et al. $[45]-1991$ & & Discrete optimal regulator & was better than a PID controller \\
\hline 18 & Lin and Lee [46]-1997 & State-space & Integral observer & simulation results showed good performance \\
\hline 19 & Previdi et al. [47]-2006 & & PID & a cost-effective alternative for volumetric pumps \\
\hline 20 & Jiang et al. $[48]-2012$ & System identification & Predictive & was better than a PID controller \\
\hline \multicolumn{5}{|c|}{ Viscosity control } \\
\hline 1 & Revesz and Hubeny [49]-1976 & & Cascade PID & used a torque sensor based viscometer and also the controller's performance was not verified \\
\hline 2 & Chiu and Lin $[50]-1998$ & ARMA & Constrained minimum variance & in-line viscometer was used which may lead to melt flow restrictions \\
\hline 3 & Chiu et al.[51]-1997, Chiu and Pong [52, 53]-1999, 2001 & System identification & Fuzzy-PID & in-line viscometer was used which may lead to melt flow restrictions, was better than a PID controller \\
\hline 4 & Kumar et al. [54]-2003 & First principle & PI & an inferential viscosity estimation was used and the controller's performance relied on its prediction accuracy \\
\hline \multicolumn{5}{|c|}{ Other control approaches } \\
\hline 1 & Stevenson [60]-1989 & Statistical approach & & no clear mention on how the controller was designed \\
\hline 2 & Yang and Lee [61, 62]-1988 & Analytical flow & PI, Feed forward & was good only for controlling long-term disturbances \\
\hline 3 & Guo et al. [63]-1993 & if-then rules and pattern recognition & PI & was capable of maintaining products' dimensional stability \\
\hline
\end{tabular}

of control schemes have been concentrated on transfer function models mostly in combination with PID approaches. Some other work has used linear time-series regression techniques in process disturbance rejection and control to achieve the process quality requirements. Such linear techniques have demonstrated some potential to reduce fluctuations in melt pressure, temperature, viscosity or any other desired variable at a fixed operating point but as the process is highly nonlinear, they are not suitable for a wide operating range which applies in industrial environments. Furthermore, the majority of the thermal control schemes are based on temperature feedback provided by wall mounted thermocouples. Therefore, it is a fundamental issue that the information provided by these thermocouples is a poor performance indicator which is highly affected by the metal wall temperature and provides no insight to the actual process thermal information. Some alternative control techniques (e.g., AI techniques) are capable of handling process nonlinearities and only a very little work can be found in the literature based on these techniques for controlling the barrel set temperatures within their desired limits. However, these did not consider about the melt temperature or melt quality control. Overall, a little work has been reported on melt temperature control based on nonlinear techniques while no work has been reported based on thermal profile measurement (i.e., rather than point/bulk measurements) techniques. Therefore, the melt thermal quality control of extrusion is in need of considerable future development to meet the existing production challenges. Perhaps, the use of the techniques such as AI in melt temperature control, individually or jointly with melt pressure may be desirable as they can handle process nonlinearities even without having exact numerical details. Likewise, it would be good to use thermal profile measurement techniques in process thermal monitoring and control rather than point/bulk techniques which are less in accuracy.

The development of viscosity control schemes has been hampered due to the lack of suitable instrumentation and severe throughput restrictions caused by the existing viscosity measuring instruments although several work attempted a number of different strategies to control the melt viscosity based on in-line rheometer measurements. Therefore, it is important to develop viscosity control strategies in polymer extrusion without involving in-line rheometer measurements or complex modifications to the machine/die. Obviously it will be a challenging task, perhaps soft sensing techniques would be able to provide some novel approaches for inferential prediction of the melt viscosity in real-time with reasonable accuracy. McAfee and McNally [76] have recently purposed a potential approach for inferential estimation of melt viscosity in polymer extrusion which may lead to develop a viscosity prediction based process control strategy.

Although, some other process control schemes (i.e., apart from the temperature, pressure or viscosity control) have been proposed with special modifications to the extruder, these are not suitable for industry due to the difficulty or unsuitability of retrofitting extruders for such modifications. Therefore, the development of new control strategies with no complex extruder modifications while having good capabilities of performing over process nonlinearities and disturbances within a wide operating window is highly important. Overall, it can be suggested that the control of the melt viscosity or melt temperature is the most appropriate approach for controlling the process melt quality [77]. However, real-time viscosity measurements may not be industrially attractive due to the possible flow restrictions. Moreover, the melt temperature control is also an indirect method of controlling the melt viscosity. Therefore, control of the melt temperature (i.e., with an advanced thermal monitoring technique rather than using a poor point/bulk technique) would be the most effective/practical way of achieving the required melt quality in polymer extrusion while serving the rapidly growing industrial needs. 


\subsection{A new approach for extrusion control}

The typical variability of the melt temperature profile across the die melt flow over different processing conditions was discussed previously by the author $[3,77,78,79,80,81,82]$ and a few other researchers $[13,17,83]$. If the melt flow is fully homogeneous in temperature, it should have flat melt temperature profile across the flow cross-section under all processing conditions (i.e., the ideal situation). However, these previous works have confirmed that the process melt temperature varies significantly across the melt flow cross-section. In fact, this information supports the argument that the most of the existing thermal control methods based on point/bulk temperature measurement feedback are not capable of controlling the temperature variations across the melt flow as these point/bulk techniques cannot accurately detect these variations. Therefore, the study of the entire melt temperature profile as a measure of the process thermal stability is more appropriate than a point/bulk measurement to ensure good quality extruded products. Unfortunately, it is quite difficult to measure a die melt temperature profile within a production environment and the most of the extruders are instrumented only with conventional wall mounted thermocouples. These are highly affected by the barrel wall temperature and also they are not capable of measuring a melt temperature profile or detecting rapid variations in melt temperature $[3,84,85]$. As alternatives to the point/bulk measurements, some of the thermal profile measurement methods (e.g., a thermocouple mesh [83], a fluorescence technique [86]) have been proposed, but these are not yet robust enough to use in a production environment due to constraints such as their complexity, limited durability, access requirements, disruptive effects on the melt flow and output, etc, although some of these techniques have been used to gather valuable process thermal information in a research setting. A detailed discussion on the existing thermal monitoring techniques in polymer extrusion was presented by the author previously [3, 77, 79]. Therefore, it is clear that process thermal monitoring in polymer extrusion is still having poor performance and hence process control techniques are also experiencing lack of performance. Here, a novel die melt temperature profile prediction soft sensor which was originally proposed by the author will provide a promising solution $[87,88]$.

In fact, it is clear that not only the level of the melt temperature but also the melt temperature variance across the melt flow have to be controlled (i.e., in parallel with the level) to achieve a better process thermal control. Therefore, the main purpose of the development of a process controller should be to select and maintain appropriate process settings to make the extruder output melt flow temperature profile as flat as possible (i.e., to achieve reduced melt temperature variations across the melt flow) while achieving the desired average melt temperature across the melt flow throughout the process operating period for a given machine and a material. As was revealed by the previous work $[13,17,79,80,81,83]$, the screw speed has a significant impact on the shape of the extruder's output melt flow temperature profile and these effects differ with the barrel set temperatures as well. Furthermore, making changes to each barrel zone temperature have different effects on the resulting melt temperature at the output [5]. The majority of the existing thermal control strategies try to manipulate the screw speed or barrel set temperatures (i.e., all zones together or individual zones) but not both at the same time. Moreover, it is quite difficult for the process operator to determine in what quantity which parameter has to be adjusted (i.e., manual process adjustments) to avoid a processing problem as making changes to one particular parameter change the effects of the all other parameters and may generate another problem due to the highly coupled nature of process variables. Therefore, a control strategy which considers all of these process variables to make control decisions would be highly effective.

In the selection of an appropriate control technique, all the above mentioned issues have to be considered as the selected control technique should be compatible with all of these requirements. Overall, the key aim is therefore to develop a process thermal control framework based on the temperature profile measurements, which manipulates the screw speed and individual barrel set temperatures together to reduce the undesirable melt temperature variations across the melt flow while maintaining the required average temperature levels. Obviously, this type of controller would have to handle complex nonlinear behaviours of the process and conventional PID approaches may not perform well over this type of situations. In this type of work, use of a control technique like fuzzy logic may be advantageous as a fuzzy logic controller (FLC) can handle process nonlinearities with a set of linguistic if-then rules which do not require exact numerical boundaries [32]. Likewise, Vlachopoulos [89] and Rauwendaal et al. [90] emphasise that the suitability of using of AI techniques (e.g., expert systems, neural networks and fuzzy logic) in the applications of polymer processing industry to overcome the existing challenges in the process modelling and control. Moreover, fuzzy logic techniques are already used in a large number of practical applications such as process control in other industries (e.g., temperature, pressure and level control; failure diagnosis and distillation column control); house hold applications like washing machines and refrigerators; motor control in power industry; various automobile, robotics and aerospace applications, etc [32]. As was discussed previously in section 1.2 , the AI techniques which were used in the extruder barrel temperature control have performed well over conventional PID controllers with a smaller overshoot and a faster settling time. Therefore, it was anticipated that fuzzy logic would suit for polymer process control as an outperforming alternative to the commonly used linear PID controllers.

\subsubsection{Fuzzy logic}

Here, a brief but a complete description is provided on the fuzzy logic controller design for the better understanding of the reader as this technique has not yet been widely used in the polymer process control. The idea of fuzzy logic was firstly proposed by professor L. A. Zadeh in the mid 1960s [91, 92]. Then, the idea of fuzzy control emerged in the early 1970s, and since then it has been becoming increasingly popular in process control as well as other control applications [32, 93, 94, 95]. Apart from the possibility of handling process nonlinearities, the other major advantages of rule based fuzzy logic controllers 
over conventional control techniques are:

(a). The tasks of the controller can be defined with multiple rules without integrating into a single analytic control law.

(b). It is simple and linguistic rules can be defined with a basic knowledge.

(c). It is not necessary to define exact numerical boundaries or limits and a fuzzy logic controller can perform with qualitative information.

(d). Low installation cost.

In general, fuzzy logic controllers are composed of four main elements: fuzzy rules (i.e., if-then linguistic rules), an inference mechanism, a fuzzification mechanism and a defuzzification mechanism. A block diagram showing a basic structure of a fuzzy logic controller (FLC) is given in Figure 2.

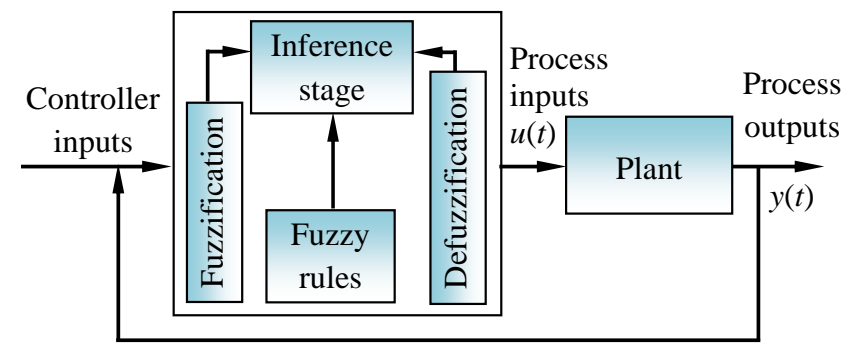

Figure 2: Basic structure of a basic fuzzy logic controller

Of the main elements of a fuzzy logic controller, the inference mechanism is extremely important. Three major inference methods are available in the literature for the fuzzy systems based on linguistic rules:

(a). Mamdani method

(b). Sugeno method

(c). Tsukamoto method

From these three methods, the first two methods are widely popular within the control field [96] where the Mamdani method [97] is the most common in practical applications [98]. In fact, one of the major differences between these methods can be recognised as the way of defining linguistic rules. An example which shows the structure of a linguistic rule in the Mamdani method is given in equation (1). Usually, the structure of a fuzzy linguistic rule includes two parts called antecedent (e.g., the first part of equation (1) before 'Then' which is relevant to the inputs) and consequent (e.g., the second part of equation (1) followed by 'Then' which is relevant to the outputs). Each rule's consequent of the Mamdani method which is defined in a similar way to equation (1) is called a fuzzy singleton. In the Sugeno method, each rule's consequent is defined as a constant, a linear function or a polynomial. In the Tsukamoto method, the consequent of each fuzzy linguistic rule is presented by a fuzzy set with a monotonic membership function which is a different way to the Mamdani and Sugeno methods. The ways of defining each rule's antecedent are usually similar in all of these methods. Basically, all of these methods have been designed to perform the same job but with different approaches while fuzzy rules and fuzzy reasoning (i.e., an inference procedure that derives conclusions from a set of fuzzy rules and other known information) are the key elements of any inference mechanism. More details on all of these inference methods can be found in the literature [98]. Moreover, a general description on the fuzzy logic controller design is presented in the following.

In the design of a rule based FLC, linguistic descriptions should map into a set of conditions known as linguistic rules. If $u_{i}$ and $y_{i}$ are the controller input and output variables respectively, a general linguistic rule for a MIMO controller can be given by equation (1).

$$
\begin{aligned}
& \text { If } u_{i, 1} \text { is } A_{i, 1} \text { and } u_{i, 2} \text { is } A_{i, 2}, \ldots, \text { and } u_{i, n} \text { is } A_{i, n} \\
& \text { Then } y_{i, 1} \text { is } B_{i, 1} \text { and } y_{i, 2} \text { is } B_{i, 2} \ldots \text {, and } y_{i, m} \text { is } B_{i, m}
\end{aligned}
$$

where $A_{i}$ and $B_{i}(i=1,2,3, \ldots, k)$ are the evaluative linguistic expressions for $i^{\text {th }}$ rule, $n$ is the number of inputs, $m$ is the number of outputs and $k$ is the total number of rules. The linguistic rule shown in equation (1) uses 'and' to link the conditions included within the rule (i.e., AND operator). Additionally, the OR or NOT operators can also be used to generate rules. In fact, a suitable operator has to be selected based on the problem specifications.

After defining of appropriate rules to represent a system, fuzzy sets are used to quantify the information defined by rule base and this is known as 'fuzzification'. The defined variables can have differing degrees of membership of sets which can vary between 0 and 1 . The whole range of fuzzy sets is known as the universe of discourse and each set within the universe of discourse is called a membership function (MF). Essentially, the designer has to determine the desired shapes of the sets and the number of sets required to define a particular variable and these selections may have some impact on the controller's performance [99]. Then, an inference mechanism should be selected to carry out two basic operations: deciding the extent of relevancy of each rule to the current situation characterised by the controller inputs known as 'matching' and drawing the appropriate conclusions to adjust inputs based on the information given by the rules known as 'inference step'. Finally, the defuzzification step is carried out which could use a method form the many strategies available such as centre of gravity and centre average (i.e., for implied fuzzy sets), max criterion, mean of maximum and centre of area (i.e., for the overall implied fuzzy sets). However, each of these methods contributes to select the final output (i.e., to combine the rules which are fired together within a particular time and to convert the information into a numerical value to perform the control action) depending on the inference technique selected. A combination of all of the above steps provides the final control action for a particular control problem.

Although fuzzy logic offers several advantages over conventional control methods, there may be some challenges as well in the use of fuzzy logic and a few of them are listed below:

- It may require prior and/or in-depth knowledge on the system in defining rules.

- It is not certain that the controller covers all the situations that can occur due to disturbances, parameter variations, noise, etc (i.e., challenges in defining accurate linguistic rules based on the available information about the process/plant). 
- It may be difficult to estimate membership functions.

- There are a number of ways of interpreting fuzzy rules, combining rules, defuzzifying output etc., and hence it may be difficult to choose the most appropriate method for a particular application.

In the design of a FLC, it is better to take all the possible actions to overcome these limitations for achieving the required/optimum performance from the controller. Further details on fuzzy logic and controller design can be found in the literature [32, 91, 92, 93, 94, 95, 96, 97, 98, 99].

\subsubsection{Model-based extrusion control with fuzzy logic}

A possible novel approach of controlling the extrusion process with dynamic process models and fuzzy logic is discussed in this section. This can be considered as a combination of model predictive and AI control techniques. Two possible arrangements of the controller under this approach are shown in Figures 3 and 4.

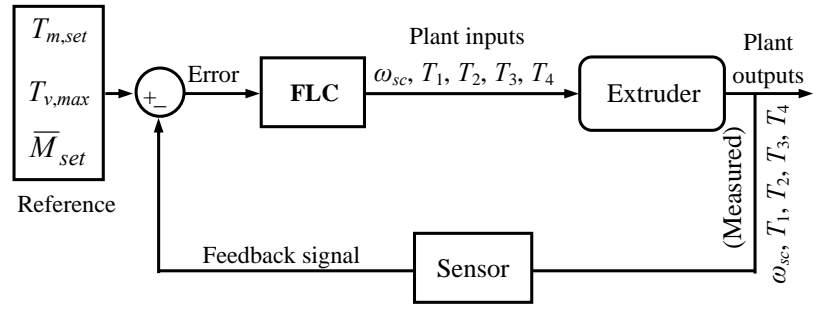

Figure 3: Extrusion control with a combination of dynamic process models and a FLC

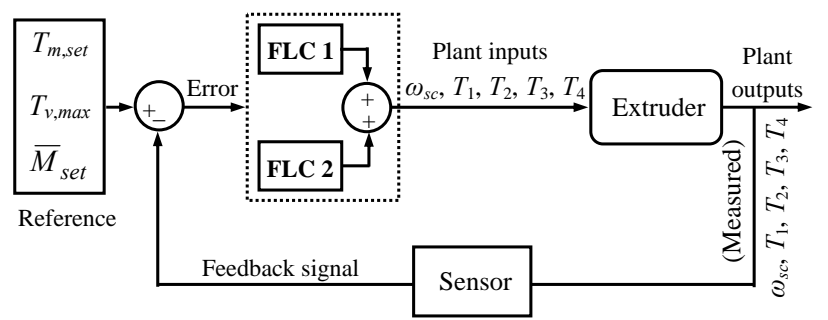

Figure 4: Extrusion control with a combination of dynamic process models and two FLCs

Here, $\omega_{s c}$ is the screw speed and $T_{1}-T_{4}$ represent the set temperatures of different barrel zones. Moreover, $T_{v, \text { max }}$ is the maximum allowable melt temperature variance across the die melt flow, $\bar{M}_{\text {set }}$ is the set/desired mass throughput rate and $T_{m, \text { set }}$ is the set/desired average melt temperature across the die melt flow. These set values can be selected carefully depending on the process scope, limitations and stability. To determine the actual values of these three variables, dynamic process models can be used if they cannot be measured to a good accuracy by using physical sensors in real-time. Then, three error signals (melt temperature variance error: $E\left(T_{v}\right)$, average melt temperature error: $E\left(T_{m, a v g}\right)$, mass throughput error: $\left.E(\bar{M})\right)$ can be generated as given in equations (2) to (4) and these can be used as the inputs to the controller.

$$
\begin{gathered}
E\left(T_{v}\right)=T_{v, a c t}-T_{v, \max } \\
E\left(T_{m, a v g}\right)=T_{m, a c t}-T_{m, s e t} \\
E(\bar{M})=\bar{M}_{a c t}-\bar{M}_{s e t}
\end{gathered}
$$

where $T_{v, a c t}$ is the actual melt temperature variance across the die melt flow, $\bar{M}_{a c t}$ is the actual mass throughput rate and $T_{m, a c t}$ is the actual average melt temperature across the die melt flow. To formulate a dynamic model to predict the temperature profile across the die melt flow, the method originally proposed by the author [80] should be useful. Here, the same model can be used to determine the melt temperature variance across the die melt flow. Likewise, other required models should also be developed based on the same modelling technique previously proposed by the author $[80,100]$. In general, the development of generalized models is highly suitable as it allows to apply the controller in a wide range of materials, machines and processing conditions

In the next stage, if-then fuzzy rules (as given by equation (1)) can be developed by combining these three errors signals simultaneously or by combining two of them together at a time. If two signals are combined together, two FLCs should be developed and the combined output of them should be taken as the plant input as shown in Figure 4. Here, a FLC or FLCs can be designed according to one of the inference methods discussed in section 2.2.1. For the development of a FLC or FLCs, shape/range of the membership functions, range of each universe of discourse (i.e., the whole range of the membership functions of each controller input and output) and the arrangement of their membership functions should be determined carefully and the relevant details can be found in the literature [98, 101, 32].

To obtain the feedback signal, a suitable physical or inferential/soft sensor, or a combination of a physical and a soft sensor can be used. Here, the major consideration should be the sensor's accuracy and its capability of providing a wide range of information about the actual melt quality. As was discussed previously, most of the existing controllers use low accuracy point/bulk temperature sensors to obtain their feedback on melt/process quality. However, it is obvious that the information provided by these sensors are limited to where they are fixed and also they are poor in detecting rapid changes in melt temperature. Therefore, it is important to use high accuracy and reliable sensor which can provide a wide range of information about the actual melt quality with a fast response time to obtain the feedback, and this would help to achieve the optimum performance from the controller. The soft sensor originally proposed by the author to predict a temperature profile across the extruder die melt flow [87, 88] should be useful in obtaining a detailed and an accurate feedback, and hence to apply with a process control strategy.

Although only three inputs have been used as the controller's inputs in this proposed approach (see Figures 3 and 4), more inputs can be added if suitable. Likewise, two or more FLCs can be used in case of adding more inputs to the controller. Here, a 
proposal was made to use fuzzy logic in extrusion control and it is highly recommended of using other AI techniques such as neuro-fuzzy and neural networks as well, for the future development of the extrusion industry. Recent work by the author proposed a new process control strategy for polymer extrusion using fuzzy logic and dynamic process models where the initial simulation results are presented [102].

\section{Conclusions}

Control approaches based on temperature, pressure and viscosity (individually or jointly) have been widely popular in extrusion control. A critical evaluation of the literature on single screw extrusion control showed that the majority of the existing control techniques show some limitations in dealing with process nonlinearities. Also, their performance have been affected by the limitations of process monitoring techniques which are poor in providing feedback on process operational/functional quality. Some control techniques may have good performance but they are not applicable in extensive industrial applications due to constraints such as melt flow restrictions, access requirements, complexity, durability, etc. Therefore, further development to the extrusion control is highly invaluable and timely to improve the efficiency of industrial processes. In this work, the importance of applying new control techniques to the extrusion control is highlighted rather than the commonly used conventional linear PID approaches. As a potential alternative, an approach of using fuzzy logic with if-then rules and dynamic process models in extrusion control is also proposed. This will provide a promising approach for extrusion control which can perform over process nonlinearities and within a wide operating window.

\section{Future Work}

In future, the proposed approach will be used to develop a novel controller for polymer extrusion control. An advanced thermal measurement technique (i.e., without using a poor point/bulk technique) which will allow to observe the actual melt quality across the melt flow will be used as the controller's feedback. Furthermore, the new controller will be tested by simulation and then it will be implemented on an industrial extruder for evaluating the performance.

\section{References}

[1] The global energy crisis,Available at: http://news.thomasnet.com/IMT/2012/04/30/the-damage-donein-transportation-which-energy-source-will-lead-to-the-greenesthighways/ [Last Accessed: 06/08/2014].

[2] A. Bredenberg, The damage done in transportation which energy source will lead to the greenest highways?Available at: http://planetforlife.com/ [Last Accessed: 06/08/2014].

[3] C. Abeykoon, Polymer Extrusion: A Study on Thermal Monitoring Techniques and Melting Issues, Lap Lambert Publishing:Verlag, 2012.

[4] C. Rauwendaal, Polymer extrusion, Hanser, 2001.

[5] M. J. Stevens, J. A. Covas, Extruder principles and operation, Chapman and Hall, 1995.

[6] P. D. Coates, In-line rheological measurements for extrusion process control, Transactions of the Institute of Measurement and Control 28 (1) (1995) 10-16.

[7] P. H. Squires, F. W. Wolf, Designing extruders for quality performance, SPE Journal 27 (4) (1971) 68-73.

[8] E. C. Brown, A. L. Kelly, P. D. Coates, Melt temperature homogeneity in single screw extrusion: effect of material type and screw geometry, SPE ANTEC Technical Papers (2004) 183-187.

[9] R. T. Fenner, Principals of polymer processing, Macmillan, 1979.

[10] C. G. Gogos, M. H. Kim, Melting phenomena and mechanism in polymer processing equipment, SPE ANTEC Technical Papers 1, paper No: 475.

[11] Y. Breaux, J.-Y. Charmeau, M. Moguedet, A simple model of throughput and pressure development for single screw, Journal of Materials Processing Technology 209 (1) (2009) 611-618.

[12] A. L. Kelly, E. C. Brown, P. D. Coates, Melt temperature field measurement in single screw extrusion: influence of melt pressure and die geometry, SPE ANTEC Technical Papers 1 (2005) 291-295.

[13] A. L. Kelly, E. C. Brown, P. D. Coates, The effect of screw geometry on melt temperature profile in single screw extrusion, Polymer Engineering and Science 46 (12) (2006) 1706-1714.

[14] K. Wilczyski, Evaluating screw performance in a single screw extruder, Polymer-Plastics Technology and Engineering 28 (7-8) (1989) 671-690.

[15] C. Rauwendaal, G. V. D. Sluis, Effect of screw surface on extruder performance, SPE ANTEC Technical Papers 1 (2004) 313-316.

[16] H. W. Gitschner, J. Lutterback, Influences of barrel wall temperature on melt throughput fluctuations in the single screw extruder, KunststoffeGerman Plastics 74 (1) (1984) 7-8.

[17] A. K. Wood, R. Rasid, Effect of process variables on melt velocity profiles in extrusion process using single screw plastics extruder, Plastics, Rubber and Composites 32 (5) (2003) 193-198.

[18] D. H. White, N. R. Schott, Dynamic testing of plastics extrusion system, SPE ANTEC Technical Papers (1972) 797-801.

[19] W. R. Fontaine, Analysis and modeling of the dynamic behavior of a plasticating extruder, Ph.D. thesis, Ohio State University, Ohio (1975).

[20] D. Fingerle, Autogenic melt temperature control system for plastic extrusion, Journal of Elastomers and Plastics 10 (4) (1978) 293-310.

[21] S. Dormeier, Digital temperature control - a way to improve the extrusion process, SPE ANTEC Technical Papers (1979) 216-219.

[22] C. Muhrer, C. Guerrero, W. I. Patterson, Extruder temperature behaviour, SPE ANTEC Technical Papers (1983) 95-98.

[23] D. Chan, L. J. Lee, Dynamic modelling of a single screw plasticating extruder, SPE ANTEC Technical Papers (1984) 77-80.

[24] D. Chan, R. W. Nelson, L. J. Lee, Dynamic behaviour of a single screw plasticating extruder part II: Dynamic modelling, Polymer Engineering \& Science 26 (20) (1986) 152-161.

[25] R. W. Nelson, D. Chan, B. Yang, L. J. Lee, Dynamic behaviour of a single screw plasticating extruder part I: Experimental study, Polymer Engineering \& Science 26 (20) (1986) 144-151.

[26] J. Parnaby, A. K. Kochhr, B. Wood, Development of computer control strategies for plastic extruders, Polymer Engineering \& Science 15 (8) (1975) 594-605.

[27] A. K. Kochhar, J. Parnaby, Dynamic modelling and control of plastics extrusion processes, Automatica 13 (2) (1977) 177-183.

[28] G. A. Hassan, J. Parnaby, Model reference optimal steady-state adaptive computer control of plastics extrusion processes, Polymer Engineering \& Science 21 (5) (1981) 276-284. 
[29] M. H. Costin, P. A. Taylor, J. D. Wright, On the dynamics and control of a plasticating extruder, Polymer Engineering \& Science 27 (17) (1982) 1095-1106.

[30] D. D. Germuska, P. A. Taylor, J. D. Wright, Adaptive and multivariable control of a single screw extrusion system, Canadian Journal of Chemical Engineering 62 (6) (1984) 790-801.

[31] E. B. Dahlin, Design and tuning digital controllers, Instruments and Control Systems 41 (6) (1968) 77-83.

[32] K. M. Passino, S. Yurkovich, Fuzzy control, Addison-Wesley, 1998.

[33] J. S. Taur, C. W. Tao, C. C. Tasi, Temperature control of a plastic extrusion barrel using pid fuzzy controllers, Int. IEEE/IAS Conference on Industrial Automation and Control: Emerging Technologies, Taipei, Taiwan (1995) 370-375.

[34] C. C. Tasi, C. H. Lu, Fuzzy supervisory predictive PID control of a plastics extruder barrel, Journal of the Chinese Institute of Engineers, 21 (5) (1998) 619-624.

[35] I. Yusuf, N. Iksan, N. S. Herman, A temperature control for plastic extruder used fuzzy genetic algorithms, International Multi Conference of Engineers and Computer Scientists, Hong Kong 2 (2010) 1075-1080.

[36] S. Ravi, P. A. Balakrishnan, Temperature response control of plastic extrusion plant using matlab/simulink, International Journal on Recent Trends in Engineering \& Technology 3 (4) (2010) 135-140.

[37] S. Ravi, P. A. Balakrishnan, Stable self tuning genetic fuzzy temperature controller for plastic extrusion system, International Journal of Reviews in Computing 5 (2011) 21-28.

[38] S. Ravi, P. A. Balakrishnan, Modelling and control of an ANFIS temperature controller for plastic extrusion process, IEEE International Conference on Communication Control \& Computing Technologies (2010) 314-320.

[39] S. Ravi, P. A. Balakrishnan, Design of synthetic optimizing neuro fuzzy temperature controller for dual screw profile plastic extruder using labview, Journal of Computer Science 7 (5) (2011) 671-677.

[40] G. Menges, M. Meissner, Improvement in extruder melt temperature control, Journal of Macromolecular Science: Part A - Chemistry A6 (3) (1972) 641-656.

[41] J. V. Leeuwen, Stock temperature measurement in plastifying equipment, Polymer Engineering \& Science 7 (2) (1967) 98-109.

[42] J. Dastych, P. Wiemer, H. Unbehauen, Robust and adaptive control of a single screw plastics extruder, Adaptive Control of Chemical Processes (ADCHEM 88) (1988) 171-176.

[43] P. Wiemer, H. Unbehauen, Decentralized discrete model reference adaptive control, Proceedings of the 10th IFAC World Congress, Munich, Germany 7 (1987) 61-66.

[44] P. K. Mercure, R. Trainor, Modelling and optimization of extruder temperature control, Computer Applications in Applied Polymer Science II - American Chemical Society (1989) 490-50638.

[45] K. C. S. Ng, W. J. B. Arden, I. G. French, Pressure control within an industrial plasticating extruder, IEEE International Conference on Control, Edinburgh, UK 1 (1991) 612-616.

[46] Y. J. Lin, G. Lee, System identification for state feedback integral observer control of polymer plastic extrusion, Polymer-Plastics Technology and Engineering 36 (5) (1997) 749-775.

[47] F. Previdi, S. M. Savaresi, A. Panarotto, Design of a feedback control system for real-time control of flow in a single-screw extruder, Control Engineering Practice 14 (9) (2006) 1111-1121.

[48] Z. Jiang, Y. Yang, S. Mo, K. Yao, F. Gao, Polymer extrusion: From control system design to product quality, Industrial \& Engineering Chemistry Research 51 (45) (2012) 14759-14770.

[49] H. Revesz, H. Hubeny, Continuous measurement and control of viscosity throughout the extrusion process, 3rd IFAC Conf. on Instrumentation \& Automation, Rubber \& Plastics Industries (1976) 69-761.

[50] S. H. Chiu, C. C. Lin, Applying the constrained minimum variance control theory on in-line viscosity control in the extrusion moulding process, Journal of Polymer Research 5 (3) (1998) 171-175.

[51] S. H. Chiu, H. C. Yiu, S. H. Pong, Development of an in-line viscometer in an extrusion molding process, Journal of Applied Polymer Science 63 (7) (2001) 919-924.

[52] S. H. Chiu, S. H. Pong, In-line viscosity control in an extrusion process with a fuzzy gain scheduled PID controller, Journal of Applied Polymer Science 74 (3) (1999) 541-555.

[53] S. H. Chiu, S. H. Pong, In-line viscosity fuzzy control, Journal of Ap- plied Polymer Science 79 (7) (2001) 1249-1255.

[54] A. Kumar, S. A. Eker, P. K. Houpt, A model based approach for estimation and control for polymer compounding, IEEE Conference on Control Applications 1 (7) (2003) 729-735.

[55] A. Pabedinskas, W. R. Cluett, Controller design and performance analysis for a reactive extrusion process, Polymer Engineering \& Science 34 (7) (1994) 585-612.

[56] T. O. Broadhead, W. I. Patterson, J. M. Dealy, Closed loop viscosity control of reactive extrusion with an in-line rheometer, Polymer Engineering \& Science 36 (23) (1996) 2840-2851

[57] S. H. Pong, C. P. Lu, M. C. Wang, S. H. Chiu, Polypropylene degradation control during reactive extrusion, Journal of Applied Polymer Science 95 (2) (2005) 280-289.

[58] S. C. Garge, M. D. Wetzel, B. A. Ogunnaike, Inference-based scheme for controlling product end-use properties in reactive extrusion process, Industrial and Engineering Chemistry Research 49 (17) (2010) 8021 8034.

[59] S. C. Garge, M. D. Wetzel, B. A. Ogunnaike, Control-relevant model identification of reactive extrusion processes, Industrial and Engineering Chemistry Research 22 (8) (2012) 1457-1467.

[60] J. F. Stevenson, Statistical procedure to identify sources of extrusion line variation, Polymer Engineering \& Science 29 (22) (1989) 1618-1627.

[61] B. Yang, L. J. Lee, Process control of profile extrusion using thermal method. part I: Mathematical modelling and system analysis, Polymer Engineering \& Science 28 (11) (1988) 697-707.

[62] B. Yang, L. J. Lee, Process control of profile extrusion using thermal method. part II: closed loop control, Polymer Engineering \& Science 28 (11) (1988) 694-717.

[63] P. Guo, X. Z. Ni, J. Zheng, Polymer extrusion production control using active recognition and adaptive control system, 2nd IEEE International Conference on Fuzzy Systems, USA 28 (11) (1993) 779-784.

[64] M. Ohshima, M. Tanigaki, Quality control of polymer production processes, Journal of Process Control 10 (2-3) (2000) 135-148.

[65] M. Kano, M. Ogawa, The state of the art in chemical process control in japan: Good practice and questionnaire survey, Journal of Process Control 20 (9) (2010) 969-982.

[66] J. Deng, K. Li, E. Harkin-Jones, M. Price, N. Karnachi, A. Kelly, J. VeraSorroche, P. Coates, E. Brown, M. Fei, Energy monitoring and quality control of a single screw extruder, Applied Energy 113 (2014) 17751785.

[67] E. G. Carrano, D. Coelho, A. Gaspar-Cunha, E. F. Wanner, R. H. Takahashi, Feedback-control operators for improved pareto-set description: Application to a polymer extrusion process, Engineering Applications of Artificial Intelligence 38 (2015) 147-167.

[68] C. Abeykoon, J. Vera-Sorroche, A. L. Kelly, E. C. Brown, P. D. Coates, J. Deng, K. Li, N. Karnachi, E. Harkin-Jones, M. Price, Process efficiency in polymer extrusion: Correlation between the energy demand and melt thermal stability, Applied Energy 135 (2014) 560-571.

[69] C. Abeykoon, J. Vera-Sorroche, A. L. Kelly, E. C. Brown, P. D. Coates, K. B. Howell, J. Deng, K. Li, N. Karnachi, E. Harkin-Jones, M. Price, Investigation of the process energy demand in polymer extrusion: A review and an experimental study, Applied Energy 136 (2014) 726-737.

[70] C. Abeykoon, A. L. Kelly, J. Vera-Sorroche, E. C. Brown, P. D. Coates, An energy saving, factory-validated disturbance decoupling control design for extrusion processes, 13th European Control Conference (ECC 2014) (2014) 1030-1035.

[71] B. K. Nguyen, G. McNally, A. Clarke, Real time measurement and control of viscosity for extrusion processes using recycled materials, Polymer Degradation and Stability 102 (2014) 212-221.

[72] L. Saerens, C. Vervaet, J. P. Remon, T. D. Beer, Process monitoring and visualization solutions for hot-melt extrusion: a review, Journal of Pharmacy and Pharmacology 66 (2013) 180-203.

[73] Q. Zheng, Z. Gao, An energy saving, factory-validated disturbance decoupling control design for extrusion processes, 10th World Congress on Intelligent Control and Automation (WCICA 2012) (2012) 2891-2896.

[74] C. Abeykoon, M. McAfee, S. Thompson, K. Li, A. L. Kelly, E. C. Brown, Investigation of torque fluctuations in extrusion through monitoring of motor variables, Proc. of 26th PPS annual Europe/Africa regional meeting, Larnaca, Cyprus, (2009) Paper No:22-O.

[75] J. Vlachopoulos, D. Strutt, Overview: Polymer processing, Materials Science and Technology 19 (2003) 1161-1169. 
[76] M. McAfee, G. McNally, Real-time measurement of melt viscosity in single-screw extrusion, Transactions of the Institute of Measurement and Control 28 (5) (2006) 481-499.

[77] C. Abeykoon, Modelling and control of melt temperature in polymer extrusion, Ph.D. thesis, Queen's University Belfast, UK (2011).

[78] C. Abeykoon, P. J. Martin, A. L. Kelley, K. Li, E. C. Brown, P. D. Coates, Investigation of the temperature homogeneity of die melt flows in polymer extrusion, Polymer Engineering and Science 54 (10) (2014) 2430 2440 .

[79] C. Abeykoon, P. J. Martin, A. L. Kelly, E. C. Brown, An evaluation of melt temperature sensors for polymer extrusion, Sensors and Actuators A: Physical 182 (2012) 16-27.

[80] C. Abeykoon, K. Li, M. McAfee, P. J. Martin, Q. Niu, A. L. Kelly, J. Deng, A new model based approach for the prediction and optimisation of thermal homogeneity in single screw extrusion, Control Engineering Practice 19 (8) (2011) 862-874.

[81] C. Abeykoon, M. McAfee, K. Li, A. L. Kelly, E. C. Brown, Monitoring the effect of operating conditions on melt temperature homogeneity in single-screw extrusion, SPE ANTEC Technical Papers 1 (2010) 1799_ 1806.

[82] C. Abeykoon, M. McAfee, K. Li, P. J. Martin, A. L. Kelly, The inferential monitoring of screw load torque to predict process fluctuations in polymer extrusion, Journal of Materials Processing Technology 211 (12) (2011) 1907-1918

[83] E. C. Brown, A. L. Kelly, P. D. Coates, Melt temperature field measurement in single screw extrusion using thermocouple meshes, Review of Scientific Instruments 75 (11) (2004) 4742-4748.

[84] X. Shen, R. Malloy, J. Pacini, An experimental evaluation of melt tempearture sensors for thermopalstic extrusion, SPE ANTEC Technical Papers (1992) 918-926.

[85] V. Schoppner, E. Enns, K. Anger, E. Giese, Dynamic temperature measurement in polymer processing, SPE ANTEC Technical Papers (2008) $228-232$.

[86] A. J. Bur, M. G. Vangel, S. C. Roth, Fluorescence based temperature measurements and applications to real-time polymer processing, Polymer Engineering and Science 41 (8) (2001) 1380-1389.

[87] C. Abeykoon, A novel soft sensor for real-time monitoring of die melt temperature profile in polymer extrusion, Patent Application Number: LK/P/1/16984.

[88] C. Abeykoon, A novel soft sensor for real-time monitoring of die melt temperature profile in polymer extrusion, IEEE Transactions on Industrial Electronics 61 (12) (2014) 7113-7123.

[89] J. Vlachopoulos, Recent progress and future challenges in computeraided polymer processing analysis and design, Report Presented at the ATV-Semapp Meeting, Funen, Denmark.

[90] C. Rauwendaal, G. Armstrong, S. Unruh, High precision extrusion lines, SPE ANTEC Technical Papers 1 (1996) 406-410.

[91] L. A. Zadeh, Fuzzy sets, Information and Control 8 (3) (1965) 338-353.

[92] L. A. Zadeh, G. J. Klir, B. Yuan, Fuzzy sets, fuzzy logic, fuzzy systems: selected papers by Lotfi A. Zadeh, World Scientific Press, 1996.

[93] H. Zhang, Why use fuzzy logic?, Available from: http://www.aptronix.com/de/whyfuzzy.htm, [Assessed 22/02/2011] (2011).

[94] A. M. F. Fileti, A. J. B. Antunes, F. V. Silva, V. Silveira, J. A. F. R. Pereira, Experimental investigations on fuzzy logic for process control, Control Engineering Practice 15 (9) (2007) 1149-1160.

[95] C. W. D. Silva, Applications of fuzzy logic in the control of robotic manipulators, Fuzzy Sets and Systems 70 (2-3) (1995) 223-234.

[96] J. S. R. Jang, C. T. Sun, E. Mizutani, Neuro-Fuzzy and soft computing: A computational approach to learning and machine intelligence, Prentice Hall, 1997.

[97] E. H. Mamdani, S. Assilian, An experiment in linguistic synthesis with a fuzzy logic controller, International Journal of Man-Machine Studies 7 (1) (1975) 1-13.

[98] J. R. Timothy, Fuzzy logic with engineering applications, 2nd Edition, Wiley-Blackwell, 2004.

[99] J. Jantzen, Design of fuzzy controllers, Technical Report: 98-E 864, Technical University of Denmark.

[100] C. Abeykoon, P. J. Martin, K. Li, A. L. Kelley, Dynamic modelling of die melt temperature profile in polymer extrusion: Effects of process settings, screw geometry and material, Journal of Applied Mathematical
Modelling 38 (4) (2014) 1224-1236.

[101] F. D. Sciascio, R. Carelli, Fuzzy basis functions for triangle-shaped membership functions: Universal approximation - MISO case, IFSA 95, Sao Paulo, Brazil 11 (5) (1995) 439-442.

[102] C. Abeykoon, A novel model-based controller for polymer extrusion, IEEE Transactions on Fuzzy Systems 22 (6). 